\title{
Hamiltonian cycles avoiding prescribed arcs in tournaments
}

\author{
Jørgen Bang-Jensen Gregory Gutin * \\ Anders Yeo \\ Department of Mathematics and Computer Science \\ Odense University, Denmark
}

\begin{abstract}
In [6], Thomassen conjectured that if $I$ is a set of $k-1$ arcs in a $k$-strong tournament $T$, then $T-I$ has a Hamiltonian cycle. This conjecture was proved by Fraisse and Thomassen [3]. We prove the following stronger result. Let $T=(V, A)$ be a $k$-strong tournament on $n$ vertices and let $X_{1}, X_{2}, \ldots, X_{l}$ be a partition of the vertex set $V$ of $T$ such that $\left|X_{1}\right| \leq\left|X_{2}\right| \leq \ldots \leq\left|X_{l}\right|$. If $k \geq \sum_{i=1}^{l-1}\left\lfloor\left|X_{i}\right| / 2\right\rfloor+\left|X_{l}\right|$, then $T-\cup_{i=1}^{l}\left\{x y \in A: x, y \in X_{i}\right\}$ has a Hamiltonian cycle. The bound on $k$ is sharp.
\end{abstract}

\section{Introduction}

In [6], Thomassen conjectured that if $I$ is a set of $k-1$ arcs in a $k$-strong tournament $T$, then $T-I$ has a Hamiltonian cycle. This conjecture was proved by Fraisse and Thomassen [3]. This result is sharp since the deletion of a set $I$ of $k$ arcs from a $k$-strong tournament may create a vertex of indegree or outdegree 0. However, the authors of [6] realized that, for some sets $I$, their bound was far from being the best possible (see, e.g., Section 5 in [6]).

*This work was supported by the Danish Research Council under grant no. 11-0534-1. The support is gratefully acknowledged. 
In this paper, we prove the following stronger result. Let $T=(V, A)$ be a $k$-strong tournament on $n$ vertices and let $X_{1}, X_{2}, \ldots, X_{l}$ be a partition of the vertex set $V$ of $T$ such that $\left|X_{1}\right| \leq\left|X_{2}\right| \leq \ldots \leq\left|X_{l}\right|$. If $k \geq$ $\sum_{i=1}^{l-1}\left\lfloor\left|X_{i}\right| / 2\right\rfloor+\left|X_{l}\right|$, then $T-\cup_{i=1}^{l}\left\{x y \in A: x, y \in X_{i}\right\}$ has a Hamiltonian cycle. The bound on $k$ is sharp (see Theorem 4.2).

It is easy to see that the above theorem by Fraisse and Thomassen follows from our result. Indeed, let $I$ be a set of arcs in a tournament $T$, let $G$ be the undirected graph obtained by ignoring all orientations of the arcs of $T\langle I\rangle$, the subgraph of $T$ which has arc set $I$ and no isolated vertices, and let $Y_{1}, \ldots, Y_{m}$ be the vertex sets of the connected components of $G$ so that $\left|Y_{1}\right| \leq \ldots \leq\left|Y_{m}\right|$. By our result, $T$ has a Hamiltonian cycle avoiding the $\operatorname{arcs}$ in $I$ if $T$ is $k$-strong, where $k \geq k^{\prime}=\sum_{i=1}^{m-1}\left\lfloor\left|Y_{i}\right| / 2\right\rfloor+\left|Y_{m}\right|$. But $k^{\prime} \leq 1+\sum_{i=1}^{m}\left(\left|Y_{i}\right|-1\right) \leq 1+\sum_{i=1}^{m} e\left(Y_{i}\right)=1+|I|$, where $e\left(Y_{i}\right)$ is the number of edges in the component of $G$ induced by $Y_{i}$.

A simple analysis of the last calculation shows precisely when the FraisseThomassen and our theorems provide the same value of strong connectivity of $T$ - namely, when $I$ consists of one tree, plus maybe some independent arcs. In all other cases our result gives a better bound. In particular, if $T\langle I\rangle$ is a union of (vertex) disjoint subtournaments of $T$ of order $n_{1}, \ldots, n_{m}$ $\left(3 \leq n_{1} \leq \ldots \leq n_{m}\right)$, then, to guarantee that $T-I$ has a Hamiltonian cycle, we need $T$ to be $\left(\sum_{i=1}^{m}\left(\begin{array}{c}n_{i} \\ 2\end{array}\right)+1\right)$-strong by the Fraisse-Thomassen theorem and to be $\left(n_{m}+\sum_{i=1}^{m-1}\left\lfloor n_{i} / 2\right\rfloor\right)$-strong by our result.

Our proof is based on Hoffmann's theorem on circulations in networks [4] and a theorem by the third author on minimal spanning 1-diregular subgraphs in semicomplete multipartite digraphs [7]. The proof of the FraisseThomassen theorem was also based on a non-trivial result, namely one from $[5]$.

\section{Terminology and notation}

We shall assume that the reader is familiar with the standard terminology on graphs and digraphs and refer the reader to [1].

By a cycle and a path in a directed graph we mean a directed simple cycle and path, respectively. Let $D$ be a digraph. $V(D)(A(D))$ denotes the vertex (arc) set of $D$. Two cycles $Q$ and $R$ (or paths) are disjoint if $V(Q) \cap V(R)=\emptyset$. A collection of vertex disjoint cycles of $D$ is called a 
cycle subgraph of $D$. A cycle subgraph $F$ of $D$ consisting of disjoint cycles $C_{1}, \ldots, C_{t}$ will be denoted $F=C_{1} \cup \ldots \cup C_{t}$. A digraph $D$ is strong if there exists a path from $x$ to $y$ and a path from $y$ to $x$ for every choice of distinct vertices $x, y$ of $D$. A digraph $D$ is $k-$ strong $(k>0)$ if $D-X$ is strong for any subset $X$ of the vertices of $D$ with $|X|<k$. Let $G$ and $H$ be subgraphs of $D$ and let $P=v_{1} v_{2} \ldots v_{k-1} v_{k}$ be a path in $D$. $P$ is called a $(G, H)$-path if $v_{1} \in V(G), v_{k} \in V(H)$ and $\left\{v_{2}, \ldots, v_{k-1}\right\} \cap(V(G) \cup V(H))=\emptyset$.

Let $C=x_{1} x_{2} \ldots x_{c} x_{1}$ be a cycle in $D$. Then we shall usually denote $x_{i+1}$ by $x_{i}^{+}$and $x_{i-1}$ by $x_{i}^{-}$, where $i=1,2, \ldots, c$ and all subscripts are taken modulo c. For a set $X$ of vertices in $C$, we denote $X^{+}=\left\{x^{+}: x \in X\right\}$ and $X^{-}=\left\{x^{-}: x \in X\right\}$. When we use such notation, the digraph $D$ considered has a spanning cycle subgraph $F$, thus every vertex $x$ of $D$ has a unique predecessor $x^{-}$and a unique succesor $x^{+}$on the unique cycle in $F$ containing $x$. The subpath of a cycle $C$ from a vertex $v$ to a vertex $w$ will be denoted by $C[v, w]$.

If $D$ has an arc $x y \in A(D)$, then we sometimes use the notation $x \rightarrow y$ and say that $x$ dominates $y$ and $y$ is dominated by $x$. For disjoint sets $X$ and $Y$ of vertices in $D$, we say that $X$ strongly dominates $Y$, and use the notation $X \Rightarrow Y$, if there is no arc from $Y$ to $X$. For arbitrary sets $X$ and $Y$ of vertices in $D,(X, Y)_{D}=\{x y \in A(D): x \in X, y \in Y\}$. In particular, if $|X|=1$, then $(X, X)_{D}$ is empty.

A digraph $H$ is called semicomplete if every two distinct vertices of $H$ are adjacent. A semicomplete digraph without cycles of length two is a tournament. Let $X_{1}, X_{2}, \ldots, X_{p}(p \geq 2)$ be a partition of the vertex set of a semicomplete digraph $H$. Then the digraph $D=H-\cup_{i=1}^{p}\left(X_{i}, X_{i}\right)_{H}$ is called semicomplete $p$-partite or semicomplete multipartite (when the value of $p$ is not important). We call $X_{1}, X_{2}, \ldots, X_{p}$ the colour classes of $D$.

\section{Preliminaries}

In this section we describe some results which will be important tools for the proof of our main result (Theorem 4.1). The following result is a very special case of a theorem proved by the third author in [7].

Theorem 3.1 Let $D$ be a semicomplete p-partite digraph with colour classes $X_{1}, X_{2}, \ldots, X_{p}$ and let $F$ be a spanning cycle subgraph of $D$ with the minimum 
possible number of cycles $t$. Then either $t=1$, or the cycles of $F$ can be labeled $C_{1}, \ldots, C_{t}$ such that the following holds: There is a pair of indices $q, q^{\prime} \in\{1,2, \ldots, p\}$ ( $q$ and $q^{\prime}$ may be equal), such that every arc to $C_{1}$ from the outside is in $\left(X_{q}^{-}, X_{q}^{+}\right)_{D}$ and every arc from $C_{t}$ to outside is in $\left(X_{q^{\prime}}^{-}, X_{q^{\prime}}^{+}\right)_{D}$.

We recall the classical theorem by Hoffmann, characterizing the existence of a feasible circulation in a network with upper and lower bounds on the arcs. Below we use the following notation. If $X$ is a subset of the vertex set of a digraph $D$, then we denote by $\bar{X}$ the set $V(D) \backslash X$. If $r$ is a function on the arc set of $D$, then $r(X, \bar{X})=\sum\{r(u v): u v \in(X, \bar{X})\}$. Let $N$ be a network. A flow $f$ in $N$ is called an integer flow if $f(a)$ is integer for every arc $a$ in $N$. A circulation is a flow of value zero in $N$.

Theorem 3.2 [4] Let $N=(V, A, \ell, c)$ denote a network with vertex set $V$, arc set $A$ and lower (upper) bound $\ell(a)(c(a))$ on every arc $a \in A$. Suppose $\ell(a), c(a)$ are non-negative integers for each $a \in A$. There exists an integer circulation $f$ in $N$ such that $\ell(a) \leq f(a) \leq c(a)$ for all $a \in A$ if and only if $c(\bar{X}, X) \geq \ell(X, \bar{X})$ for all proper subsets $X$ of $V$.

For a proof of this theorem, see for example [2, page 50]. We finish this section by the following simple but useful lemma. The proof is left for the reader.

Lemma 3.3 Let $T$ be a tournament, and let $Y_{1}, Y_{2}, \ldots, Y_{s}(s \geq 1)$ be disjoint sets of vertices in $T$ and let $x$ and $y$ be arbitrary distinct vertices in $V(T)-$ $\left(Y_{1} \cup Y_{2} \cup \ldots \cup Y_{s}\right)$. If there exist $k$ disjoint $(x, y)$-paths in $T$, then there exist at least $k-\sum_{i=1}^{s}\left\lfloor\left|Y_{i}\right| / 2\right\rfloor$ disjoint $(x, y)$-paths in $T-\cup_{i=1}^{s}\left(Y_{i}, Y_{i}\right)$.

\section{Results}

Theorem 4.1 Let $T=(V, A)$ be a $k$-strong tournament on $n$ vertices, and let $X_{1}, X_{2}, \ldots, X_{l}(l \geq 1)$ be a partition of $V\left(V=\cup_{i=1}^{l} X_{i}, X_{i} \cap X_{j}=\emptyset\right.$ for every $i \neq j)$. Set $D=T-\cup_{i=1}^{l}\left(X_{i}, X_{i}\right)_{T}, D_{i}=D \cup\left(X_{i}, X_{i}\right)_{T}, D_{i, j}=D \cup$ $\left(X_{i}, X_{i}\right)_{T} \cup\left(X_{j}, X_{j}\right)_{T}(1 \leq i, j \leq l)$. If $1 \leq\left|X_{1}\right| \leq\left|X_{2}\right| \leq \ldots \leq\left|X_{l}\right| \leq n / 2$ and $k \geq\left|X_{l}\right|+\sum_{i=1}^{l-1}\left\lfloor\left|X_{i}\right| / 2\right\rfloor$, then the following hold.

(a) If $x \in X_{i}$ and $y \in X_{j}(1 \leq i \neq j \leq l)$, then there are $\left\lfloor\left|X_{i}\right| / 2\right\rfloor+$ $\left\lfloor\left|X_{j}\right| / 2\right\rfloor+\left\lceil\left|X_{l}\right| / 2\right\rceil$ disjoint $(x, y)$-paths in $D_{i, j}$. 
(b) If $x, y \in X_{i}(x \neq y)$, then there are $\left|X_{i}\right|$ disjoint $(x, y)$-paths in $D_{i}$. Furthermore there is an $(x, y)$-path in $D$.

(c) D is strong.

(d) D contains a spanning cycle subgraph.

(e) D is Hamiltonian.

\section{Proof:}

Claim (a) easily follows from Lemma 3.3 .

Proof of Claim (b): From Lemma 3.3 we easily get that there are $\left|X_{i}\right|$ disjoint $(x, y)$-paths in $D_{i}$. By deleting all arcs in $\left(X_{i}, X_{i}\right)_{T}$, we can destroy at most $\left|X_{i}\right|-1$ of these paths, since at most $\left|X_{i}\right|-2$ paths can contain vertices from $X_{i}-\{x, y\}$, and only one path can be the arc $x y$. Thus there is an $(x, y)$-path in $D$.

Proof of Claim (c): Let $x$ and $y$ be arbitrary distinct vertices of $D$. If $\{x, y\} \subseteq X_{i}$, then there is an $(x, y)$-path in $D$, because of Claim (b). Therefore we may now assume that $x \in X_{i}$ and $y \in X_{j}$, where $i \neq j$. Let $W$ be the maximal set of vertices such that for all $w \in W$, there is an $(x, w)$-path in $D$.

Assume that $W \cap X_{j} \neq \emptyset$ and let $w \in W \cap X_{j}$. From (b) there is a $(w, y)$-path in $D$. Now we see that there is a $(x, y)$-path in $D$.

Assume that $W \cap X_{j}=\emptyset$. Therefore, there is no $(x, y)$-path in $D_{j}$, and at most $\left|X_{i}\right|-1$ disjoint $(x, y)$-paths in $D_{i, j}$, since each $(x, y)$-path in $D_{i, j}$ must include a vertex from $X_{i}-x$. This is a contradiction against (a), since $\left\lfloor\left|X_{i}\right| / 2\right\rfloor+\left\lceil\left|X_{l}\right| / 2\right\rceil \geq\left|X_{i}\right|>\left|X_{i}\right|-1$.

Hence we have proved that there exists a $(x, y)$-path in $D$ for an arbitrary choice of distinct $x$ and $y$, which means that $D$ is strong.

Proof of Claim (d): Let $D^{\prime}=\left(V^{\prime} \cup V^{\prime \prime}, A\left(D^{\prime}\right)\right)$ be the digraph obtained from $D$ by replacing each vertex $v \in V$ by two vertices $v^{\prime}$ and $v^{\prime \prime}$ joined by an arc from $v^{\prime}$ to $v^{\prime \prime}$. For each original arc $u v \in A, D^{\prime}$ contains the $\operatorname{arc} u^{\prime \prime} v^{\prime}$.

Let $N=\left(V^{\prime} \cup V^{\prime \prime}, A(N), \ell, c\right)\left(A(N)=A\left(D^{\prime}\right)\right)$ be the network obtained from $D^{\prime}$ by specifying the following lower and upper bounds for the arcs. Every arc of the kind $u^{\prime \prime} v^{\prime}$ (corresponding to an original arc in $D$ ) has lower 
bound zero and an upper bound $\infty$. Every $\operatorname{arc} x^{\prime} x^{\prime \prime}$ where $x \in V$ has lower and upper bounds equal to one.

For a subset $B$ of $V, B^{\prime}$ and $B^{\prime \prime}$ will stand for the sets $\left\{v^{\prime}: v \in B\right\}$ and $\left\{v^{\prime \prime}: v \in B\right\}$, respectively.

It is easy to see that every feasible integer circulation in $N$ corresponds to a spanning cycle subgraph in $D$ and vice versa. Hence, by Theorem 3.2 , it suffices to prove that for every proper subset $U$ of $V^{\prime} \cup V^{\prime \prime}$ we have $c(\bar{U}, U) \geq \ell(U, \bar{U})$.

Assume that $c(\bar{U}, U)<\ell(U, \bar{U})$, where $U$ is a proper subset of $V^{\prime} \cup V^{\prime \prime}$. The vertex set $V$ of $D$ can be partitioned into the following sets: $Y=\{y \in$ $\left.V: y^{\prime} \in U, y^{\prime \prime} \in \bar{U}\right\}, Z=\left\{z \in V: z^{\prime \prime} \in U, z^{\prime} \in \bar{U}\right\}, R_{1}=\{v \in V:$ $\left.\left\{v^{\prime}, v^{\prime \prime}\right\} \subseteq U\right\}$ and $R_{2}=\left\{v \in V:\left\{v^{\prime}, v^{\prime \prime}\right\} \subseteq \bar{U}\right\}$. We have $|Z|<|Y|$ since $|Z| \leq c(\bar{U}, U)$ and $\ell(U, \bar{U})=|Y|$.

Observe that an arc $u v \in A(D)$ between two vertices in $Y \subseteq V(D)$ will correspond to an arc $u^{\prime \prime} v^{\prime}$ in $N$ with $u^{\prime \prime} \in \bar{U}$ and $v^{\prime} \in U$, thus contributing $\infty$ to $c(\bar{U}, U)$. Therefore there is an $i \in\{1,2, \ldots, l\}$ such that $Y \subseteq X_{i}$. If $D$ has an arc $y r_{1}$ from $Y$ to $R_{1}$, then $y^{\prime \prime} r_{1}^{\prime} \in A(N)$ contributes $\infty$ to $c(\bar{U}, U)$. Hence, $R_{1} \Rightarrow Y$. Analogously, $\left(R_{1} \cup Y\right) \Rightarrow R_{2}$. Since $|Z|<|Y| \leq\left|X_{i}\right| \leq n / 2$ and $Y \subseteq X_{i}$, we obtain that either $W_{1}=R_{1}-X_{i}$ or $W_{2}=R_{2}-X_{i}$ is nonempty. Since $X_{1}, \ldots, X_{l}$ form a partition of $V(D)$, there is $j, j \neq i$, so that $\left(W_{1} \cup W_{2}\right) \cap X_{j} \neq \emptyset$.

Assume that $\left|W_{1} \cap X_{j}\right| \geq\left|W_{2} \cap X_{j}\right|$ and choose a vertex $w_{1}$ in $W_{1} \cap X_{j}$ and vertex $y \in Y$. Set $S=\left(W_{2} \cap X_{j}\right) \cup Z \cup\left(X_{i}-Y\right)$. By (a) there exist at least $\left|X_{i}\right|+\left\lfloor\left|X_{j}\right| / 2\right\rfloor$ disjoint $\left(y, w_{1}\right)$-paths in $D_{i, j}$. However, $D_{i, j}-S$ has no $\left(y, w_{1}\right)$-paths and $|S| \leq\left\lfloor\left|X_{j}\right| / 2\right\rfloor+|Z|+\left|X_{i}\right|-|Y|<\left\lfloor\left|X_{j}\right| / 2\right\rfloor+\left|X_{i}\right|$; a contradiction.

If $\left|W_{1} \cap X_{j}\right|<\left|W_{2} \cap X_{j}\right|$, then choose a vertex $w_{2}$ in $W_{2} \cap X_{j}$ and vertex $y \in Y$. Set $S=\left(W_{1} \cap X_{j}\right) \cup Z \cup\left(X_{i}-Y\right)$. Now $D_{i, j}-S$ has no $\left(w_{2}, y\right)$-path and we obtain a contradiction as above.

Proof of (e): Assume that $D$ is not Hamiltonian.

We first observe that $D$ is a semicomplete multipartite digraph with colour classes $X_{1}, X_{2}, \ldots, X_{l}$. By (c) the digraph $D$ is strong. By (d) $D$ contains a spanning cycle subgraph. Let $F$ be a spanning cycle subgraph of $D$ with the minimal possible number of cycles $t$. Assume that $t \geq 2$. Let $C_{1}, \ldots, C_{t}$ be a labeling of the cycles of $F$ determined in Theorem 3.1. By Theorem 3.1, there is a pair of indices $q, q^{\prime} \in\{1,2, \ldots, p\}$ such that the conclusion of the 
theorem holds. Let us fix the labeling and pair of indices above.

If $\left|V\left(C_{1}\right)\right|>n / 2$, we reverse all the arcs in $D$ and relabel the cycles in the spanning cycle subgraph corresponding to $F$. Hence, we may assume that $\left|V\left(C_{1}\right)\right| \leq n / 2$. Moreover assume that $\left|V\left(C_{1}\right)\right|$ has the minimal possible value. Set $W=V\left(C_{2}\right) \cup \ldots \cup V\left(C_{t}\right)$. For all $i=1,2, \ldots, l$ set $z_{i}=\mid V\left(C_{1}\right) \cap$ $X_{i} \mid$.

Assume that $z_{q}=\left|V\left(C_{1}\right)\right| / 2$. There are $\left|V\left(C_{1}\right)\right|\left(\left|V\left(C_{1}\right)\right|-1\right) / 2$ arcs in $\left(V\left(C_{1}\right), V\left(C_{1}\right)\right)_{T}$, and, by Theorem 3.1, there are at most $z_{q}\left(\left|X_{q}\right|-z_{q}\right)$ $\operatorname{arcs}$ in $\left(W, V\left(C_{1}\right)\right)_{D}$. Let $i \in\{1, \ldots, l\}$. Since $z_{i} /\left|V\left(C_{1}\right)\right| \leq 1 / 2$, we have $\left|\left(X_{i} \cap W, X_{i} \cap V\left(C_{1}\right)\right)_{T}\right| \leq z_{i}\left(\left|X_{i}\right|-z_{i}\right) \leq\left|V\left(C_{1}\right)\right|\left\lfloor\left|X_{i}\right| / 2\right\rfloor$. Now we obtain the following:

$$
\begin{aligned}
\left|\left(V, V\left(C_{1}\right)\right)_{T}\right| & \leq\left|V\left(C_{1}\right)\right|\left(\left|V\left(C_{1}\right)\right|-1\right) / 2+z_{q}\left(\left|X_{q}\right|-z_{q}\right)+\sum_{i=1}^{l} z_{i}\left(\left|X_{i}\right|-z_{i}\right) \\
& \leq\left|V\left(C_{1}\right)\right|\left(\left|V\left(C_{1}\right)\right|-1\right) / 2+2 z_{q}\left(\left|X_{q}\right|-z_{q}\right)+\sum_{\substack{i=1 \\
i \neq q}}^{l}\left|V\left(C_{1}\right)\right|\left\lfloor\left|X_{i}\right| / 2\right\rfloor \\
& =\left|V\left(C_{1}\right)\right|\left[\left|V\left(C_{1}\right)\right| / 2-1 / 2+\left|X_{q}\right|-\left|V\left(C_{1}\right)\right| / 2+\sum_{\substack{i=1 \\
i \neq q}}^{l}\left\lfloor\left|X_{i}\right| / 2\right\rfloor\right] \\
& \left.<\left|V\left(C_{1}\right)\right|\left[\left|X_{q}\right|+\sum_{\substack{i=1 \\
i \neq q}}^{l}|| X_{i} \mid / 2\right\rfloor\right] .
\end{aligned}
$$

This implies that there is a vertex $w$ in $V\left(C_{1}\right)$ such that

$$
\left|(V, w)_{T}\right|<\left|X_{q}\right|+\sum_{i=1, i \neq q}^{l}\left\lfloor\left|X_{i}\right| / 2\right\rfloor \leq k,
$$

which is a contradiction against the fact that $T$ is $k$-strong. Therefore we have shown that $\left|V\left(C_{1}\right) \cap X_{q}\right|<\left|V\left(C_{1}\right)\right| / 2$.

Let

$$
S=\left\{s \in V\left(C_{1}\right): \text { there exists an arc from } W \text { to } s^{+} \text {in } D\right\} .
$$

Note that, by Theorem 3.1, $S \subseteq X_{q} \cap V\left(C_{1}\right)$. Since $\left|V\left(C_{1}\right) \cap X_{q}\right|<$ $\left|V\left(C_{1}\right)\right| / 2$, the set $R=V\left(C_{1}\right)-\left(X_{q} \cup X_{q}^{+}\right)$is not empty. We prove that $R \Rightarrow S$ in $D$. Assume that there exist $s \in S$ and $r \in R$ so that $s r \in A(D)$. There is vertex $w \in V\left(C_{i}\right)$ (for some $i, 2 \leq i \leq l$ ) so that $w s^{+} \in A(D)$. Since $w^{+} \in X_{q}$ and $r^{-} \notin X_{q}$, the arc $r^{-} w^{+} \in A(D)\left(w^{+} r^{-} \notin A(D)\right.$ because $w^{++} \notin X_{q}$ ). Replace the cycles $C_{1}$ and $C_{i}$ by the cycles $C_{1}[r, s] r$ and $C_{i}\left[w^{+}, w\right] C_{1}\left[s^{+}, r^{-}\right] w^{+}$in $F$. The new spanning cycle subgraph $F^{\prime}$ has $t$ cycles as well. However, the first cycle of $F^{\prime}$ contains less vertices than $C_{1}$ does; a contradiction. 
Let $X_{j}$ be a colour class of $D$ so that $X_{j} \cap R$ is not empty. Let $r$ be a vertex in $X_{j} \cap R$ and let $w_{q}$ be a vertex in $X_{q} \cap W$. Consider two cases.

Case 1: $S$ is a proper subset of $X_{q} \cap V\left(C_{1}\right)$.

Let $x \in\left(X_{q} \cap C_{1}\right)-S$. For all colour classes, $X_{i} \neq X_{q}$ we have $\mid X_{i} \cap$ $V\left(C_{1}\right)|>| X_{i} \cap W \mid$ because of the following. Assume that there is a colour class, $X_{i} \neq X_{q}$, with $\left|X_{i} \cap V\left(C_{1}\right)\right| \leq\left|X_{i} \cap W\right|$. Let $w \in X_{i} \cap W$ be arbitrary. Claim (a) implies that there exist $\left\lfloor\left|X_{i}\right| / 2\right\rfloor+\left|X_{q}\right|$ disjoint $(w, x)$-paths in $D_{i, q}$. However, $B=\left[\left(W \cap X_{q}\right) \cup S^{+}\right] \cup\left(X_{i} \cap V\left(C_{1}\right)\right)$ separates $x$ from $w$ in $D_{i, q}$ and $|B|<\left|X_{q}\right|+\left\lfloor\left|X_{i}\right| / 2\right\rfloor ;$ a contradiction.

Since $\left|V\left(C_{1}\right)\right| \leq|V(D)| / 2$, we obtain $\left|X_{q} \cap V\left(C_{1}\right)\right| \leq\left|X_{q} \cap W\right|$. Claim (a) implies that there exist $\left\lfloor\left|X_{j}\right| / 2\right\rfloor+\left|X_{q}\right|$ disjoint $\left(w_{q}, r\right)$-paths in $D_{j, q}$. However, $B=\left[\left(V\left(C_{1}\right) \cap X_{q}\right) \cup S^{+}\right] \cup\left(X_{j} \cap W\right)$ separates $r$ from $w_{q}$ in $D_{j, q}$ and $|B|<\left|X_{q}\right|+\left\lfloor\left|X_{j}\right| / 2\right\rfloor$; a contradiction.

Case 2: $S=X_{q} \cap V\left(C_{1}\right)$.

Subcase 2a: $\left|X_{j} \cap V\left(C_{1}\right)\right| \geq\left|X_{j} \cap W\right|$ or $|S| \leq\left|X_{q} \cap W\right|$.

Set $B=S^{+} \cup\left(X_{j} \cap W\right)$. The digraph $D_{j, q}-B$ has no $\left(w_{q}, r\right)$-paths since $R \Rightarrow S$ in $D$. If $\left|X_{j} \cap V\left(C_{1}\right)\right| \geq\left|X_{j} \cap W\right|$, then $|B|<\left\lfloor\left|X_{j}\right| / 2\right\rfloor+\left|X_{q}\right|$ since $X_{q} \cap W$ is not empty. If $|S| \leq\left|X_{q} \cap W\right|$, then $|B|<\left\lfloor\left|X_{q}\right| / 2\right\rfloor+\left|X_{j}\right|$ since $X_{j} \cap V\left(C_{1}\right)$ is not empty. Each of the last two bounds for $|B|$ contradicts (a).

Subcase 2b: $\left|X_{j} \cap V\left(C_{1}\right)\right|<\left|X_{j} \cap W\right|$ and $|S|>\left|X_{q} \cap W\right|$.

If $X_{j} \cap W \subseteq\left(X_{q} \cap W\right)^{-}$, then set $B=S^{+} \cup\left(X_{j} \cap W\right)$. The digraph $D_{j, q}-B$ has no $\left(w_{q}, r\right)$-path. However, $|B| \leq\left|X_{q}\right|<\left\lfloor\left|X_{j}\right| / 2\right\rfloor+\left|X_{q}\right|$; a contradiction to (a).

If $\left(X_{j} \cap W\right)-\left(X_{q} \cap W\right)^{-} \neq \emptyset$, then set $B=\left(X_{q} \cap W\right) \cup\left(X_{q} \cap W\right)^{-} \cup\left(X_{j} \cap\right.$ $V\left(C_{1}\right)$ ). The digraph $D_{j, q}-B$ has no $(w, x)$-path, where $x \in V\left(C_{1}\right) \cap X_{q}$ and $w \in\left(X_{j} \cap W\right)-\left(X_{q} \cap W\right)^{-}$. However, $|B|<\left\lfloor\left|X_{j}\right| / 2\right\rfloor+\left|X_{q}\right|$; a contradiction to (a).

Hence, we have got a contradiction in both cases which implies that $D$ is Hamiltonian.

The bound for $k$ in Theorem 4.1 is sharp because of the following theorem. 
Theorem 4.2 Let $2 \leq r_{1} \leq r_{2} \leq \ldots \leq r_{l}$ be arbitrary integers, then there exists a tournament $T$ and a collection $X_{1}, X_{2}, \ldots, X_{l}$ of disjoint sets of vertices in $T$ such that

(i) $T$ is $\left(r_{l}-1+\sum_{i=1}^{l-1}\left\lfloor r_{i} / 2\right\rfloor\right)$-strong;

(ii) $\left|X_{i}\right|=r_{i}$ for $i=1,2, \ldots, l$;

(iii) $D=T-\cup_{i=1}^{l}\left(X_{i}, X_{i}\right)_{T}$ is not Hamiltonian.

Proof: Let $k=r_{l}+\sum_{i=1}^{l-1}\left\lfloor r_{i} / 2\right\rfloor$. We construct a tournament $T$ with the properties (i)-(iii): $V(T)=X_{1} \cup X_{2} \cup \ldots \cup X_{l} \cup Y_{1}^{\prime} \cup Y_{2}^{\prime} \cup Y_{1}^{\prime \prime} \cup Y_{2}^{\prime \prime} \cup Z$, where all the sets in the union are mutually disjoint. Let $\left|Y_{1}^{\prime}\right|=\left|Y_{2}^{\prime}\right|=\left|Y_{1}^{\prime \prime}\right|=$ $\left|Y_{2}^{\prime \prime}\right|=k,|Z|=r_{l}-1, X_{j}=\left\{x_{j 1}, \ldots, x_{j, r_{j}}\right\}, X_{j}^{\prime}=\left\{x_{j 1}, \ldots, x_{j,\left\lfloor r_{i} / 2\right\rfloor}\right\}$ and $X_{j}^{\prime \prime}=$ $\left\{x_{j,\left\{r_{i} / 2\right\rfloor+1}, \ldots, x_{j, r_{j}}\right\}$ for every $j \in\{1, \ldots, l-1\}$. Set $S^{\prime}=\left(\cup_{j=1}^{l-1} X_{j}^{\prime}\right) \cup Y_{1}^{\prime} \cup Y_{2}^{\prime}$, $S^{\prime \prime}=\left(\cup_{j=1}^{l-1} X_{j}^{\prime \prime}\right) \cup Y_{1}^{\prime \prime} \cup Y_{2}^{\prime \prime}$. The arc set of $T$ is defined as follows. Let $S^{\prime} \Rightarrow X_{l} \Rightarrow S^{\prime \prime} \Rightarrow Z \Rightarrow S^{\prime}$. If $s^{\prime} \in S^{\prime}$ and $s^{\prime \prime} \in S^{\prime \prime}$, then $s^{\prime} \rightarrow s^{\prime \prime}$ unless there exists $j \in\{1, \ldots, l-1\}$ so that both $s^{\prime}$ and $s^{\prime \prime}$ are from $X_{j}$ in which case $s^{\prime \prime} \rightarrow s^{\prime}$. For every $i=\left\{{ }^{\prime},{ }^{\prime \prime}\right\}$ and $j \in\{1, \ldots, l-1\}, X_{j}^{i} \Rightarrow Y_{1}^{i} \Rightarrow Y_{2}^{i} \Rightarrow X_{j}^{i}$. The direction of the arcs between the vertices non-adjacent so far can be chosen arbitrary.

To see that $T$ is $(k-1)$-strong, note that the deletion of any $k-2$ vertices leaves at least one vertex $y_{i}^{j}$ in each of the sets $Y_{i}^{j}$, and either (a) a vertex $z$ in $Z$, or (b) an edge $s^{\prime \prime} \rightarrow s^{\prime}$ from some $X_{j}^{\prime \prime}$ to the corresponding $X_{j}^{\prime}$. In case (a), there is a cycle $z y_{1}^{\prime} y_{2}^{\prime} y_{1}^{\prime \prime} y_{2}^{\prime \prime} z$ remaining, and in case (b) we have the cycle $s^{\prime} y_{1}^{\prime} y_{2}^{\prime} y_{1}^{\prime \prime} y_{2}^{\prime \prime} s^{\prime \prime} s^{\prime}$. In either case, every other vertex sends and receives at least one edge to/from the cycle, so the remaining digraph is strong.

Assume that $D=T-\cup_{i=1}^{l}\left(X_{i}, X_{i}\right)_{T}$ is Hamiltonian. In a Hamiltonian cycle of $D$, after every visit to $X_{l}$, the cycle must pass through $Z$ before returning to $X_{l}$ since $A(D-Z) \subseteq\left(S^{\prime}, S^{\prime \prime}\right)_{T} \cup\left(S^{\prime}, X_{l}\right)_{T} \cup\left(X_{l}, S^{\prime \prime}\right)_{T}$. However, there are more vertices of $X_{l}$ than of $Z$, thus there is no Hamiltonian cycle.

\section{Conclusions and open problems}

In fact, this paper is concerned with aspects of the following general question. Which sets $B$ of edges of the complete graph $K_{n}$ have the property that every $k$-strong orientation of $K_{n}$ induces a Hamiltonian digraph on $K_{n}-B$ ? The 
Fraisse-Thomassen theorem says that this is the case whenever $A$ contains at most $k-1$ edges. Here, it has been shown that a union of disjoint cliques of sizes $r_{1}, \ldots, r_{l}$ has the property, whenever $\sum_{i=1}^{l}\left\lfloor r_{i} / 2\right\rfloor+\max _{1 \leq i \leq l}\left\{\left\lceil r_{i} / 2\right\rceil\right\} \leq$ $k$. This is the best possible result for unions of cliques. Also, it implies the Fraisse-Thomassen theorem.

It seems natural to investigate bounds for $k$ in different cases of the set $B$. In particular, what are sharp bounds for $k$ when $B$ is a spanning forest of $K_{n}$ consisting of $m$ disjoint paths containing $r_{1}, \ldots, r_{m}$ vertices, respectively ? The same question can be asked if we replace "paths" by "stars" or by "cycles" (in the last case "spanning forest" should also be replaced by "spanning cycle subgraph").

\section{Acknowledgments}

We are grateful to the referee for numerous remarks and suggestions that have improved the presentation.

\section{References}

[1] J.A. Bondy and U.S.R. Murty, Graph Theory with Applications, MacMillan Press, 1976.

[2] L. R. Ford and D. R. Fulkerson, Flows in Networks, Princeton University Press, Princeton New Jersey (1962).

[3] P. Fraisse and C. Thomassen, Hamiltonian dicycles avoiding prescribed arcs in tournaments. Graphs and Combin. 3 (1987) 239-250.

[4] A. J. Hoffmann, Some recent applications of the theory of linear inequalities to extremal combinatorial analysis, Proc. Symposia on Applied Math. 10 (1960) 113-127.

[5] C. Thomassen, Hamiltonian-connected tournaments. J. Combin. Theory B28 (1980) 142-163.

[6] C. Thomassen, Edge-disjoint Hamiltonian paths and cycles in tournaments. Proc. London Math. Soc. 45 (1982) 151-168. 
[7] A. Yeo, One-diregular subgraphs in semicomplete multipartite digraphs. To appear in J. Graph Theory. 